\title{
Dietary intake, nutritional status and mental wellbeing in street homeless and hostel residents in Reading
}

\author{
R. Fallaize, J.V. Seale, C. Mortin, L. Armstrong and J.A. Lovegrove \\ The Hugh Sinclair Unit of Human Nutrition, University of Reading, Reading, RG6 6AP, UK
}

Homelessness, which includes individuals sleeping rough (on the streets) or in hostels/temporary accommodation, increased $34 \%$ between 2011 and $2014^{(1)}$. Homeless adults have greater physical and mental health needs and elevated levels of acute and chronic disease $^{(2)}$. Although malnutrition and wasting have been reported in the homeless ${ }^{(3)}$, the specific nutritional issues faced by each homeless community remain unclear. Food availability is a major problem for street homeless individuals and whilst some hostels provide meals, these may not adhere to national dietary recommendations.

The aim of this study was to compare the dietary intake, nutritional status and wellbeing of street homeless and hostel residents in Reading (UK).

Street homeless ( $n$ 24) and hostel residents ( $n$ 52) were recruited from the Reading region. Dietary intake was determined using the European Prospective Investigation into Cancer and Nutrition (EPIC) Norfolk Food Frequency Questionnaire (FFQ). The Patient Health Questionnaire: Somatic Anxiety Depressive Symptoms (PHQ-SADS) assessed for signs indicative of mental illness. Demographic, behavioural and physiological information was collected using closed-ended questions and anthropometric measurements. General linear models were adjusted for age, gender and ethnicity.

Street homeless and hostel residents were aged 38 (SD 10.4) and 38 (SD 10.5) years with a body mass index of $23 \mathrm{~kg} / \mathrm{m}^{2}$ and $25 \mathrm{~kg} / \mathrm{m}^{2}$ respectively $(P=0.371)$ Reported intakes of saturated fat (SFA) $(15.0 \%$ TE vs. $13.6 \% \mathrm{TE}, P=0.039)$, milk $(449 \mathrm{~g}$ vs. $229 \mathrm{~g}, P=0.005)$ and potato (107 g vs. $63 \mathrm{~g}, P=0.021)$ were all significantly higher in hostel residents compared with street homeless, although street homeless individuals had a higher intake of soups and sauces ( $83 \mathrm{~g}$ vs. $48 \mathrm{~g}, P=0.002)$. Additionally, daily intakes of SFA exceeded, and non-starch polysaccharide (NSP) (13.4 g, SD 7.9) and fruit and vegetables (F\&V) (3.7 portions) failed to meet government recommendations and that reported in the general population $\left(12.6 \%\right.$ SFA, $13.7 \mathrm{~g} \mathrm{NSP}, 4 \cdot 1$ portions F\&V) ${ }^{(4)}$. In total, $73 \%$ of homeless adults reported having enough to eat, with the majority consuming 2 meals a day $(n 35)$. Handgrip strength $(P=0.028)$ and PHQ9 scores $(P=$ 0.011) were significantly higher in street homeless individuals than hostel residents, with depression (PHQ-9) reaching a 'moderate' classification.

Homeless individuals demonstrated poorer dietary intakes than the general population, with street homeless at a significant disadvantage. Interventions to improve dietary intake are urgently needed, although may need to be targeted to the specific needs of the homeless groups.

1. Medcalf P \& Russell GK (2014) Clin Med 14(4), 349-353.

2. Hwang SW (2000) JAMA 283, 2152-2157.

3. Langnäse K \& Müller MJ (1999) Public Health Nutr 4, 805-811.

4. Bates B, Lennox A, Prentice A et al. (2014) Public Health England 2014051. 